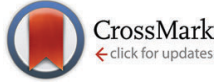

Cite this: J. Mater. Chem. C, 2016, 4, 4630

Received 4th April 2016

Accepted 5th May 2016

DOI: $10.1039 / c 6 t c 01360 k$

www.rsc.org/MaterialsC

\section{A dual-phase architecture for efficient amplified spontaneous emission in lead iodide perovskites $\dagger$}

\author{
Stefanie Neutzner, ${ }^{\mathrm{ab}}$ Ajay Ram Srimath Kandada, ${ }^{* \mathrm{a}}$ Guglielmo Lanzani $^{\mathrm{ab}}$ and \\ Annamaria Petrozza*a
}

\begin{abstract}
Multi-particle Auger interactions are one of the major intrinsic limiting factors for lasing applications of lead halide perovskites. In this communication, we report a possible way to tackle this problem by investigating the transfer of the photo-excited population to radiative defect inclusions using transient absorption (TA) spectroscopy. We demonstrate a reduction of the threshold of amplified spontaneous emission (ASE) in the presence of an efficient transfer.
\end{abstract}

\section{Introduction}

Hybrid lead halide perovskites have recently emerged as exceptional materials for different optoelectronic applications. Beyond the very good performances demonstrated when embodied in photovoltaic (PV) devices, ${ }^{3,4}$ high photoluminescence quantum yields, long carrier lifetimes $^{5-7}$ and wavelength tunability ${ }^{8,9}$ place them as good candidates for lasing applications. Recently, amplified spontaneous emission (ASE) as well as lasing have been demonstrated in a variety of sample morphologies ranging from polycrystalline thin films ${ }^{10}$ single crystals, ${ }^{11}$ nanowires ${ }^{12,13}$ to films made from colloidal nanocrystals. ${ }^{14}$ The reported gain thresholds are very promising; however, they have been achieved under ultra-short $(\mathrm{fs} / \mathrm{ns})^{2}$ pulse excitation. Therefore operation under technologically relevant continuous wave $(\mathrm{cw})$ conditions or under electrical injection remains a major challenge in the field.

Within the high excitation density regime needed for population inversion in semiconductors, non-radiative Auger recombination leads to a population loss, heating of the material and higher gain threshold. ${ }^{1}$ One of the established routes implemented in quantum well lasers to counter this process is to create a controlled phasearchitecture of spatially separated domains for pumping and population inversion. Such engineered material systems enable

${ }^{a}$ Center for Nano Science and Technology@PoliMi, Istituto Italiano di Tecnologia, via Giovanni Pascoli 70/3, 20133, Milan, Italy.E-mail: srinivasa.srimath@iit.it, annamaria.petrozza@iit.it

${ }^{b}$ Dipartimento di Fisica, Politecnico di Milano, Piazza L. da Vinci, 32, 20133 Milano, Italy

$\dagger$ Electronic supplementary information (ESI) available. See DOI: 10.1039/c6tc01360k spatial redistribution of the excited state population such that inter-particle Auger interactions are lowered, thus leading to an overall reduction in the gain threshold..$^{15-17}$

Here, we report a proof of principle for the implementation of such an architecture by the exploitation of radiative defect inclusions in polycrystalline thin films of methyl-ammonium lead iodide $\left(\mathrm{MAPbI}_{3}\right)$ perovskites. These defects form after the phase transition of the semiconductor, from the room temperature (RT) tetragonal structure to the orthorhombic one, stable at temperatures below $160 \mathrm{~K} .^{18,19}$ We use transient absorption spectroscopy to show an efficient transfer of carriers from the dominant orthorhombic phase of the semiconductor to these defects, which intrinsically represents the spatial separation of pumping and emission domains. We demonstrate that this leads to a reduction of the gain threshold and allows us to retrieve design parameters for an optimized architecture. This result provides an interesting route to the defect engineering of these self-assembled materials for cw lasing applications.

\section{Results}

Fig. 1a shows the optical absorption spectra of a poly-crystalline film of $\mathrm{MAPbI}_{3}$ deposited on glass, taken at different temperatures (see Fig. S1, ESI $\dagger$ for structural data). As previously reported, ${ }^{20,21}$ the orthorhombic phase, stable at low temperature $(<160 \mathrm{~K})$, has a larger gap compared to that of the RT phase. The optical edges are placed at $740 \mathrm{~nm}$ and $760 \mathrm{~nm}$ at $150 \mathrm{~K}$ and $290 \mathrm{~K}$ respectively, which then red-shift as the temperature is lowered as a result of continuous expansion/shrinkage of the lattice. ${ }^{22}$ In a single crystal, the phase transition occurs at $160 \mathrm{~K}^{23}$ Importantly, it has been shown often that polycrystalline films do not exhibit a sharp transition, at least with regard to the optical signature of the phases. ${ }^{24-26}$ This is evidenced in Fig. 1a by the existence of the spectral signature of the tetragonal phase in the absorption spectra even down to $140 \mathrm{~K}$. Similar trends are observed also in the photoluminescence spectra shown in Fig. 1b. The lower energy emission peak initially blue-shifts in 

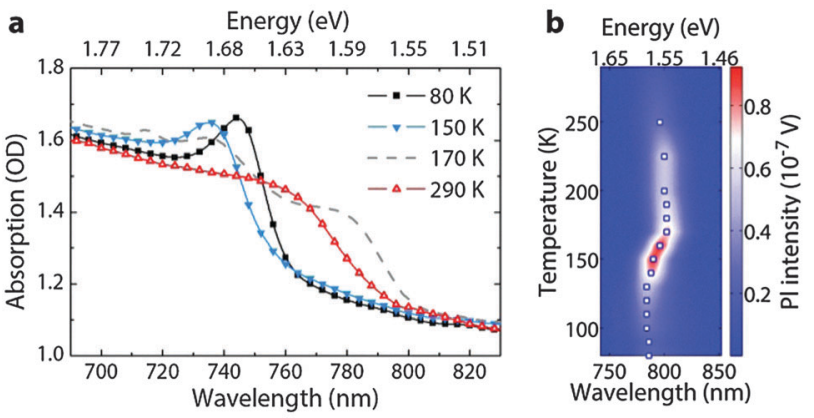

Fig. 1 (a) Temperature dependence of the absorption edge. (b) Spectral evolution of the low energy photoluminescence $(\mathrm{PL})$ peak as a function of temperature. The white squares mark the position of the PL maximum as a guide to the eye.

the temperature range of 170 to $130 \mathrm{~K}$, passing through a maximum in emission intensity and thereafter red-shifts. The nature of the lower energy emission feature in the anomalous temperature range has already been debated in previous reports and assigned either to a bound-exciton next to free-exciton emission, ${ }^{10,23}$ therefore belonging to the same structural phase, or to a coexistence of the tetragonal and orthorhombic phases. ${ }^{24,25}$ Recently, Kong et al. have shown temperature dependent X-ray diffraction studies of the film, ${ }^{25}$ demonstrating the coexistence of the tetragonal and orthorhombic phases within a range of $30 \mathrm{~K}$ around the phase transition temperature. However, a very low density of such intrusions can still be optically perceived at temperatures well below such range (i.e. $77 \mathrm{~K}$, see the $\mathrm{ESI} \dagger$ of this manuscript). Thus, Kong et al. suggested, following the theoretical prediction of Yin et al., ${ }^{27}$ that these are inter- band gap states of the orthorhombic phase due to iodide and lead vacancies, which form radiative recombination centres. We can also speculate that these correspond to linear defect sites, which form in between crystalline grains of the orthorhombic phase. Though, there is a need for a detailed investigation to identify the nature of these defects, from now on, we will generally indicate them as colour centres.

To quantitatively assess the photo-carrier transfer from the semiconductor bandgap to the colour centres, we performed fs-TA spectroscopy at different temperatures with the pump at $530 \mathrm{~nm}$. In Fig. 2, we show the TA spectra of $\mathrm{MAPbI}_{3}$ taken at $150 \mathrm{~K}$ (for other temperatures, compare Fig. S2, ESI $\dagger$ ). The main features are the photo-bleach (PB) bands due to state filling by the photo-excited carriers. ${ }^{21}$ In particular, we observe two $\mathrm{PB}$ bands, peaking at $730 \mathrm{~nm}$ (labeled $\left.\mathrm{PB}_{\mathrm{O}}\right)$ and $760 \mathrm{~nm}\left(\mathrm{~PB}_{\mathrm{D}}\right)$, corresponding to the orthorhombic phase and the defect phase respectively. The time evolution of the spectra shows quenching of $\mathrm{PB}_{\mathrm{O}}$ and a concomitant growth in the $\mathrm{PB}_{\mathrm{D}}$ band in tens of picoseconds (see dynamics in Fig. 2c). Fitting the dynamics at $150 \mathrm{~K}$ of the two peaks with simple exponential decays, including one term accounting for the transfer time, delivers a time constant of about $13 \pm 2$ ps for the transfer (Fig. 2c).

Note that such a transfer can be observed even at lower temperatures, down to $77 \mathrm{~K}$, albeit with a lower amplitude and not only at the phase transition, as claimed earlier. ${ }^{28}$ Since the efficiency of the process decreases at lower temperatures (the transfer rate and the strength of the transfer component decrease with lowering of the temperature, compare Fig. S3b, ESI $\dagger$ ), we assume that this is related to a change in the density of colour centres.

To further substantiate our hypothesis of population transfer versus other explanations, such as slow de-trapping and to

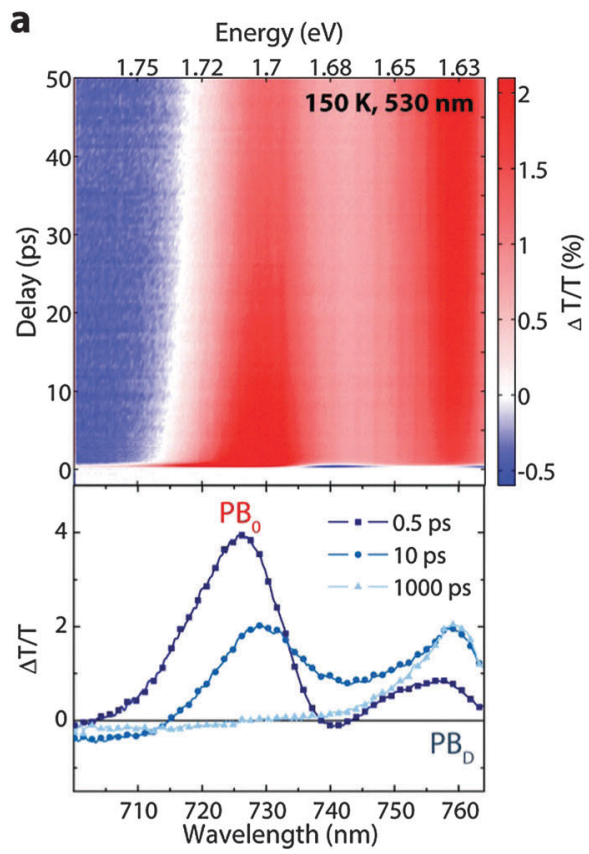

b
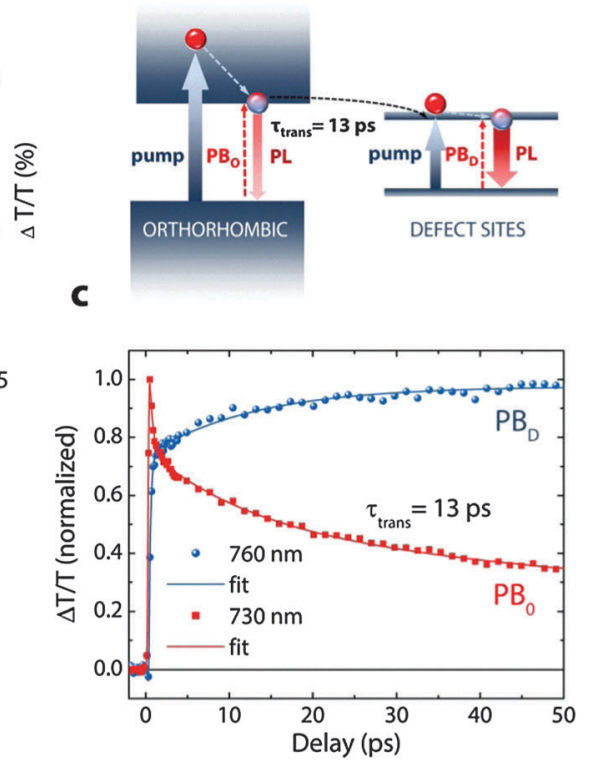

Fig. 2 (a) Top: Spectral evolution of the TA signal within 50 ps. Bottom: TA-spectra representing three different time steps. (b) Sketch illustrating the observed processes. (c) Time evolution and corresponding fits for the two PB signals at $150 \mathrm{~K}$. 

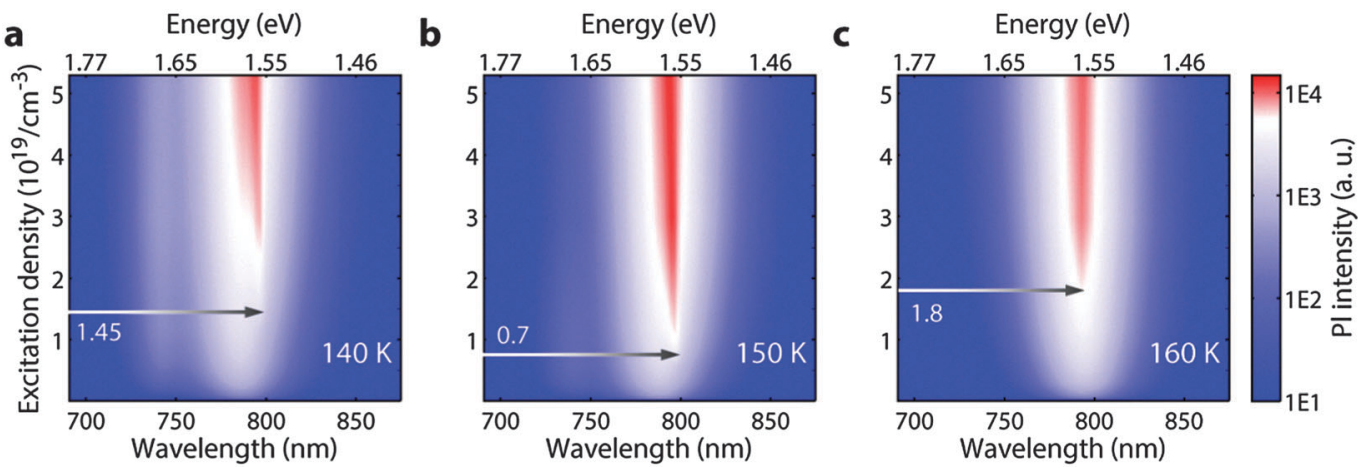

Fig. 3 Steady state PL measurements as a function of excitation density for $140 \mathrm{~K}$ (a) $150 \mathrm{~K}$ (b) and $160 \mathrm{~K}$ (c). The corresponding ASE threshold is marked for each temperature.

exclude contribution from overlapping TA features, we performed a TA experiment with two different pump wavelengths, above and below the orthorhombic phase energy. Pumping at $400 \mathrm{~nm}$, very similar to the previous case, we photo-excite both the phases and we observe the increase of $\mathrm{PB}_{\mathrm{D}}$ due to the transfer of population in the ps timescale (Fig. S4, ESI $\dagger$ ). Upon pumping at $760 \mathrm{~nm}$, we do not observe any $\mathrm{PB}_{\mathrm{O}}$ feature and we just observe the recombination dynamics of $\mathrm{PB}_{\mathrm{D}}$. This unambiguously proves our assignment of carrier transfer and supports the presence of a defined dual domain structure for pumping and emission.

In order to test the effectiveness of this system for lowering the threshold of lasing, we performed steady state emission measurements with fs-excitation at different temperatures to analyze the ASE thresholds. Since our aim here is to investigate the possible effect of population transfer, we performed the measurements at $140 \mathrm{~K}, 150 \mathrm{~K}$ and $160 \mathrm{~K}$, all within the phase transition regime, as shown in Fig. 3. This small range guarantees immunity of the observed behavior to temperature induced effects and ensures a critical density of colour centres to observe a perceivable change. As shown in Fig. 3, we find that the ASE thresholds at $140 \mathrm{~K}$ and $160 \mathrm{~K}$ are at $1.45 \times 10^{19}$ and $1.8 \times 10^{19}$ respectively, while it is lowered to $7 \times 10^{18}$ at $150 \mathrm{~K}$. This is supported by the fact that the transfer is most efficient at $150 \mathrm{~K}$ as observed in the TA experiment (see Fig. S3, ESI $\dagger$ ).

\section{Discussion}

Our result points to the possibility of energy relocation from the carrier plasma to radiative defects states, thereby reducing the effect of non-radiative Auger losses. This occurs by exploiting a dual phase architecture that is naturally engineered within the crystalline semiconductor. To optimize such a process, with the aim of transferring the acquired know-how to a system which can operate at RT, we need to deduce design principles. The important parameter here is the transfer rate, along with the relative defect density. The main purpose of the transfer process is to replenish any loss in the population due to Auger, thus mitigating the loss in population inversion needed for the gain mechanism. If the transfer time were much faster than the Auger mechanism within the low energy phase, this would result in the loss of even the transferred population. On the other hand, it should be faster than the recombination within the high energy phase. Thus a precise engineering of the transfer rate is extremely important.

We observe that the transfer rate is diffusion limited and thus correlated with the orthorhombic domain size and the density of the colour centres. This is evident from the temperature dependence of the rate, with the transfer becoming slower at lower temperatures along with a concomitant reduction in the amplitude of the transfer component of the PB dynamics (see comparison of the transfer dynamics in Fig. S3, ESI $\dagger$ ). Assuming a mobility $(\mu)$ of $50 \mathrm{~cm}^{2} \mathrm{~V}^{-1} \mathrm{~s}^{-1}$ reported for these materials at $150 \mathrm{~K},{ }^{29}$ and with the observed transfer time ( $\left.\tau_{\text {trans }}\right)$ of $13 \mathrm{ps}$, we can estimate the effective length travelled by the carriers before they are quenched, $L_{\mathrm{D}}(n)=\sqrt{\tau_{\text {trans }} \cdot \frac{\mu k_{\mathrm{b}} T}{e}}$, to be around $29 \mathrm{~nm}$. This represents the effective length-scale of the orthorhombic phase within the materials. At lower temperatures, at $120 \mathrm{~K}$, where the relative concentration of colour centres is lower (thus larger domains of the orthorhombic phase), we observe a transfer time of around $60 \mathrm{ps}$, corresponding to an effective diffusion of about $60 \mathrm{~nm}$ before the carriers can be transferred. Moreover, in a sample that is composed of smaller crystallites and hence is prone to a higher density of grain boundaries, the transfer times are much faster due to the reduction of domain size. Thus effective domain sizes have to be suitably engineered to obtain an efficient transfer process.

\section{Conclusions}

In summary, we have investigated the interaction between the orthorhombic phase and radiative defect states in $\mathrm{MAPbI}_{3}$ below the structural phase transition temperature using TA spectroscopy. We unambiguously assign the previously observed abnormal emission behaviour to transfer between the orthorhombic phase and defect sites and demonstrate that an efficient transfer can lower the threshold for ASE. Our results suggest fundamental design principles for mixed phase architectures for lasing applications, based on the interplay between Auger and transfer rates as well as the distribution and size of the respective domains. 


\section{Acknowledgements}

The authors thank Marina Gandini for the preparation of the samples. Funding from the EU Horizon 2020 Research and Innovation program under the grant agreement No. 643238 (SYNCHRONICS) and Fondazione Cariplo (IPER-LUCE, 2015-0080) is acknowledged.

\section{Notes and references}

1 M. Saba, M. Cadelano, D. Marongiu, F. Chen, V. Sarritzu, N. Sestu, C. Figus, M. Aresti, R. Piras, A. Geddo Lehmann, C. Cannas, A. Musinu, F. Quochi, A. Mura and G. Bongiovanni, Nat. Commun., 2014, 5, 5049.

2 M. Cadelano, V. Sarritzu, N. Sestu, D. Marongiu, F. Chen, R. Piras, R. Corpino, C. M. Carbonaro, F. Quochi, M. Saba, A. Mura and G. Bongiovanni, Adv. Opt. Mater., 2015, 3, 1557-1564.

3 H. Zhou, Q. Chen, G. Li, S. Luo, T.-b. Song, H.-S. Duan, Z. Hong, J. You, Y. Liu and Y. Yang, Science, 2014, 345, 542-546.

4 N. J. Jeon, J. H. Noh, W. S. Yang, Y. C. Kim, S. Ryu, J. Seo and S. Il Seok, Nature, 2015, 517, 476-480.

5 C. Wehrenfennig, G. E. Eperon, M. B. Johnston, H. J. Snaith and L. M. Herz, Adv. Mater., 2014, 26, 1584-1589.

6 S. D. Stranks, G. E. Eperon, G. Grancini, C. Menelaou, M. J. P. Alcocer, T. Leijtens, L. M. Herz, A. Petrozza and H. J. Snaith, Science, 2013, 342, 341-344.

7 V. D'Innocenzo, A. R. Srimath Kandada, M. De Bastiani, M. Gandini and A. Petrozza, J. Am. Chem. Soc., 2014, 136, 17730-17733.

8 E. T. Hoke, D. J. Slotcavage, E. R. Dohner, A. R. Bowring, H. I. Karunadasa and M. D. McGehee, Chem. Sci., 2015, 6, 613-617.

9 S. Pathak, N. Sakai, F. Wisnivesky Rocca Rivarola, S. D. Stranks, J. Liu, G. E. Eperon, C. Ducati, K. Wojciechowski, J. T. Griffiths, A. A. Haghighirad, A. Pellaroque, R. H. Friend and H. J. Snaith, Chem. Mater., 2015, 27, 8066-8075.

10 G. Xing, N. Mathews, S. S. Lim, N. Yantara, X. Liu, D. Sabba, M. Grätzel, S. Mhaisalkar and T. C. Sum, Nat. Mater., 2014, 13, 476-480.

11 Q. Zhang, S. T. Ha, X. Liu, T. C. Sum and Q. Xiong, Nano Lett., 2014, 14, 5995-6001.
12 Y. Fu, H. Zhu, A. W. Schrader, D. Liang, Q. Ding, P. Joshi, L. Hwang, X.-Y. Zhu and S. Jin, Nano Lett., 2016, 16, 1000-1008.

13 H. Zhu, Y. Fu, F. Meng, X. Wu, Z. Gong, Q. Ding, M. V Gustafsson, M. T. Trinh, S. Jin and X. Zhu, Nat. Mater., 2015, 14, 636-642.

14 S. Yakunin, L. Protesescu, F. Krieg, M. I. Bodnarchuk, G. Nedelcu, M. Humer, G. De Luca, M. Fiebig, W. Heiss and M. V Kovalenko, Nat. Commun., 2015, 6, 8056.

15 A. V. Uskov, Y. Boucher, J. Le Bihan and J. McInerney, Appl. Phys. Lett., 1998, 73, 1499-1501.

16 O. Gauthier-Lafaye, P. Boucaud, F. H. Julien, S. Sauvage, S. Cabaret, J.-M. Lourtioz, V. Thierry-Mieg and R. Planel, Appl. Phys. Lett., 1997, 71, 3619.

17 F. H. Julien, A. Saár and J. Wang, Electron. Lett., 1995, 31, 838-839.

18 C. C. Stoumpos, C. D. Malliakas and M. G. Kanatzidis, Inorg. Chem., 2013, 52, 9019-9038.

19 T. Baikie, Y. Fang, J. M. Kadro, M. Schreyer, F. Wei, S. G. Mhaisalkar, M. Gratzel and T. J. White, J. Mater. Chem. A, 2013, 1, 5628.

20 V. D’Innocenzo, G. Grancini, M. J. P. Alcocer, A. R. S. Kandada, S. D. Stranks, M. M. Lee, G. Lanzani, H. J. Snaith and A. Petrozza, Nat. Commun., 2014, 5, 3586.

21 G. Grancini, A. R. Srimath Kandada, J. M. Frost, A. J. Barker, M. De Bastiani, M. Gandini, S. Marras, G. Lanzani, A. Walsh and A. Petrozza, Nat. Photonics, 2015, 9, 695-701.

22 Y. P. Varshni, Physica, 1967, 34, 149-154.

23 H. H. Fang, R. Raissa, M. Abdu-Aguye, S. Adjokatse, G. R. Blake, J. Even and M. A. Loi, Adv. Funct. Mater., 2015, 25, 2378-2385.

24 C. Wehrenfennig, M. Liu, H. J. Snaith, M. B. Johnston and L. M. Herz, APL Mater., 2014, 2, 081513.

25 W. Kong, Z. Ye, Z. Qi, B. Zhang, M. Wang, A. Rahimi-Iman and H. Wu, Phys. Chem. Chem. Phys., 2015, 17, 16405-16411.

26 K. Wu, A. Bera, C. Ma, Y. Du, Y. Yang, L. Li and T. Wu, Phys. Chem. Chem. Phys., 2014, 16, 22476-22481.

27 W.-J. Yin, T. Shi and Y. Yan, Appl. Phys. Lett., 2014, 104, 063903.

28 H. Wang, L. Whittaker-Brooks and G. R. Fleming, J. Phys. Chem. C, 2015, 119, 19590-19595.

29 R. L. Milot, G. E. Eperon, H. J. Snaith, M. B. Johnston and L. M. Herz, Adv. Funct. Mater., 2015, 25, 6218-6227. 